05;06.4

\title{
Генерация сверхизлучения импульсным механическим воздействием
}

\author{
(ㄷ А.И. Александров, В.Г. Шевченко, И.А. Александров \\ Институт синтетических полимерных материалов им. Н.С. Ениколопова РАН, Москва, Россия \\ E-mail: alivaleksandr@mail.ru
}

Поступило в Редакцию 19 ноября 2019 г.

В окончательной редакции 19 ноября 2019 г.

Принято к публикации 15 января 2020 г.

\begin{abstract}
Установлен эффект импульсной механической активации радиочастотного сверхизлучения в параметрическом режиме: при воздействии внешнего реологического взрыва под высоким давлением на парамагнитный композит - систему скрещенных спинов на основе биядерных комплексов $\mathrm{Co}(\mathrm{QH})_{2}-\mathrm{O}-\mathrm{Co}(\mathrm{QH})_{2}$ или $\mathrm{Mn}(\mathrm{QH})_{2}-\mathrm{O}-\mathrm{Mn}(\mathrm{QH})_{2}(\mathrm{QH}$ - лиганд на основе 3,6-ди-трет-бутилпирокатехина) в полистирольной матрице. Предложены возможный метод обработки данных по импульсному механическому воздействию и вероятная схема возникновения эффекта.
\end{abstract}

Ключевые слова: сверхизлучение, парамагнитный композит, реологический взрыв.

DOI: 10.21883/PJTF.2020.07.49220.18119

Сверхизлучение (СИ) [1] и эффект Дзялошинского-Мория [2,3] - фундаментальные физические явления, которые известны довольно давно. Ранее в полимерных композитах $\mathrm{PS}+\mathrm{Co}(\mathrm{acac})_{2}+2 \mathrm{QH}_{2}$ и $\mathrm{PS}+\mathrm{Mn}(\mathrm{acac})_{2}+2 \mathrm{QH}_{2}$ (PS - полистирол, $\mathrm{Co}(\mathrm{acac})_{2}$ и $\mathrm{Mn}(\mathrm{acac})_{2}$ - ацетилацетонаты кобальта и марганца, $\mathrm{QH}_{2}$ - 3,6-ди-трет-бутилпирокатехин) при реализации процесса сверхбыстрого разрушения под высоким давлением (реологического взрыва (РВ) [4]) регистрировались импульсы радиочастотного сверхизлучения [5]. Также было установлено, что данные композиты проявляют мультиферроидные свойства и в них наблюдается эффект Дзялошинского-Мория за счет образования биядерных комплексов кобальта (БК Со) или марганца (БК Mn) [6].

Цель настоящей работы - выяснить возможность реализации параметрического режима радиочастотного СИ (когда при воздействии внешнего реологического взрыва под высоким давлением возникает импульс сверхизлучения в образце, в котором исключен процесс реологического взрыва), а также предложить метод обработки данных по импульсному механическому воздействию и вероятную схему возникновения эффекта.

Композиты были получены введением $\mathrm{Co}(\mathrm{QH})_{2}$ $\left(\right.$ или $\left.\mathrm{Mn}(\mathrm{QH})_{2}\right)$ (синтез по известной методике [7], $\mathrm{QH}$ - лиганд на основе 3,6-ди-трет-бутилпирокатехина) в матрицу PS таким образом, чтобы при этом в $1 \mathrm{~cm}^{3}$ композита возникало $N$ БК Со (или БК $\mathrm{Mn}$ ) $\left(N=0.1 \cdot 10^{20}, 0.2 \cdot 10^{20}, 0.3 \cdot 10^{20}, 0.4 \cdot 10^{20}, 0.5 \cdot 10^{20}\right.$, $\left.0.6 \cdot 10^{20}, 0.7 \cdot 10^{20}\right)$. Исследуемые образцы представляли собой таблетки диаметром $14 \mathrm{~mm}$ и толщиной $0.2 \mathrm{~mm}$. Это исключало протекание процесса реологического взрыва в образцах вплоть до $\sim 6 \mathrm{GPa}$.

Для исследования процессов возникновения импульсов сверхизлучения при механическом воздействии внешним РВ использовалась специальная ячейка высокого давления, которая помещалась в машину сжатия
ИС-500 (рис. 1,a). Внешний РВ проводился быстрым одноосном сжатием образцов чистого полистирола 1 диаметром $10 \mathrm{~mm}$ и толщиной $2 \mathrm{~mm}$ при одном и том же давлении $(\sim 2 \mathrm{GPa})$ и скорости сдавливания $0.5 \mathrm{GPa} / \mathrm{s}$. Ячейка (рис. 1,a) изолирована от прессового оборудования и состоит из наковальни Бриджмена 2, стальной обоймы 3 и пуансонов 4, изолированных от обоймы, между которыми находится исследуемый образец 5. Цифрой 6 на рис. 1, $a$ обозначена изоляция. Пуансоны через сопротивление нагрузки $50 \Omega$ соединены с двухканальным цифровым осциллографом Tektronix MSO 200 (7 на рис. 1,a). Ячейка позволяет регистрировать переменный ток $J(t)$, генерируемый электрической составляющей $E(t)$ электромагнитного излучения, возникающего в образце.

Процесс СИ - это процесс когерентного спонтанного излучения, интенсивность которого $\sim N^{2}$, где $N-$ число центров возбуждения. Интенсивность сверхизлучения как функция времени описывается формулой Дике [1]:

$$
I(t)=I_{0}\left(\frac{N+1}{2}\right)^{2} \operatorname{sech}^{2}\left[\frac{N+1}{2 T}\left(t-t_{0}\right)\right],
$$

$I(t)$ описывает импульс СИ, который достигает максимума при $t=t_{0}$. Величина максимума пропорциональна $N^{2}, t_{0}$ - время задержки импульса, $t_{0}=\tau_{c} \ln N, \tau_{c}-$ длительность импульса.

В данном исследовании регистрировался переменный ток $J(t)$, генерируемый электрической составляющей $E(t)$ электромагнитного излучения, которое возникает в исследуемых образцах при механическом воздействии. Поскольку $J(t) \sim E(t)$, а интенсивность электромагнитного излучения $I(t) \sim[E(t)]^{2}$, имеем $I(t) \sim[J(t)]^{2}$ (на рис. $1, b$ приведены характерные импульсы $I(t)$ и их аппроксимация по формуле Дике). Установлено, что интенсивность пиков $I\left(t_{0}\right)$ (при $t=t_{0}$ ) пропорциональна квадрату концентрации БК $N^{2}$. Это хорошо 

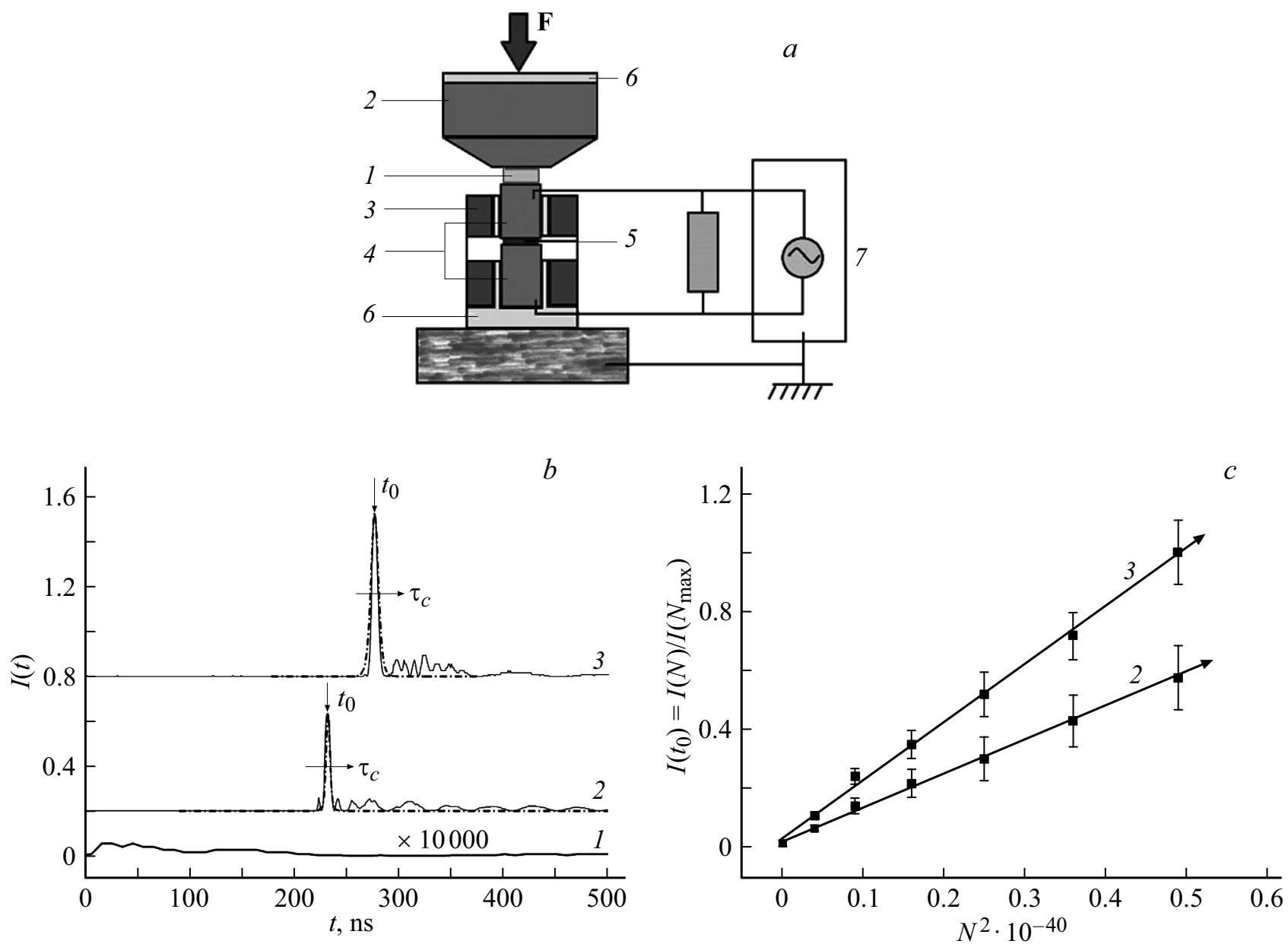

Рис. 1. $a$ - схема ячейки высокого давления (пояснение в тексте); $b$ - временна́я развертка нормированных сигналов $I(t) \sim[E(t)]^{2}$ для PS-матрицы $(1)$ и образцов, содержащих БК Со $(2)$ и БК $\mathrm{Mn}(3) ; c$ - нормированная зависимость $I\left(t_{0}\right)$ от квадрата концентрации комплексов в $1 \mathrm{~cm}^{3}$ композита для образцов, содержащих БК Со (2) и БК $\mathrm{Mn}(3)$.

видно из рис. 1, $c$, на котором показана зависимость нормированной амплитуды $I\left(t_{0}\right)=I(N) / I\left(N_{\max }\right)$ от $N^{2}$ для БК кобальта и марганца (в качестве $N_{\max }$ взято максимальное значение для БК марганца). Форма линии полос излучения также соответствует закону (1), характерному для процессов СИ: экспоненциальный симметричный подъем и спад (штрихпунктир на рис. $1, b)$. Для того чтобы установить, какие молекулярные структуры ответственны за процессы СИ, были проведены ЭПР-исследования стабильных радикальных продуктов в системах $\mathrm{PS}+\mathrm{Co}(\mathrm{QH})_{2}$ или $\mathrm{PS}+\mathrm{Mn}(\mathrm{QH})_{2}$. Далее по известной методике [8] был проведен компьютерный анализ и рассчитаны теоретические спектры ЭПР путем многопараметрического решения квантового релаксационного уравнения, характеризующегося гамильтонианом

$$
\begin{aligned}
\mathscr{H}= & g_{a} \beta H S^{a}+g_{b} \beta H S^{b}+J S^{a} S^{b}+G\left[S^{a} S^{b}\right] \\
& +D\left[S^{a} S^{b}-3 S_{z}^{a} S_{z}^{b}\right]+E\left(S_{x}^{a} S_{x}^{b}-S_{y}^{a} S_{y}^{b}\right) \\
& +A_{a, i s o} I^{a} S^{a}+A_{b, i s o} I^{b} S^{b},
\end{aligned}
$$

где $\beta-$ магнетон Бора, $H-$ напряженность магнитного поля, $S$ и $I-$ квантовые числа спина электрона и ядра соответственно, $g_{a}=\left(g_{a x}+g_{a y}+g_{a z}\right) / 3$, $g_{b}=\left(g_{b x}+g_{b y}+g_{b z}\right) / 3-g$-факторы, $A_{a, i s o}=\left(A_{a x}+\right.$ $\left.+A_{a y}+A_{a z}\right) / 3, A_{b, \text { iso }}=\left(A_{b x}+A_{b y}+A_{b z}\right) / 3$ - константы сверхтонкого магнитного взаимодействия, $J$ и $G$ константы изотропного и анизотропного обменного взаимодействия, $D$ и $E-$ константы диполь-дипольного и спин-спинового взаимодействия.

Компьютерный анализ показал, что для экспериментальных спектров ЭПР можно рассчитать теоретический спектр только при условии, что анализируемый методом ЭПР объект состоит из двух неэквивалентных парамагнитных центров $a$ и $b$, связанных между собой обменным взаимодействием, т.е. является комплексом, состоящим из двух парамагнитных центров - БК. Расчет показал, что для центра БК Со (или БК Mn) $g$-факторы, константы сверхтонкого взаимодействия и ширины соответствующих линий ЭПР равны: $g_{a x}=1.9426$ (1.9165), $g_{a y}=1.9958$ (1.9625), $g_{a z}=2.0900$ (1.8633), $A_{a x}=1.47$ (4.34) mT, $A_{a y}=1.85$ (8.19) mT, $A_{a z}=1.19$ 

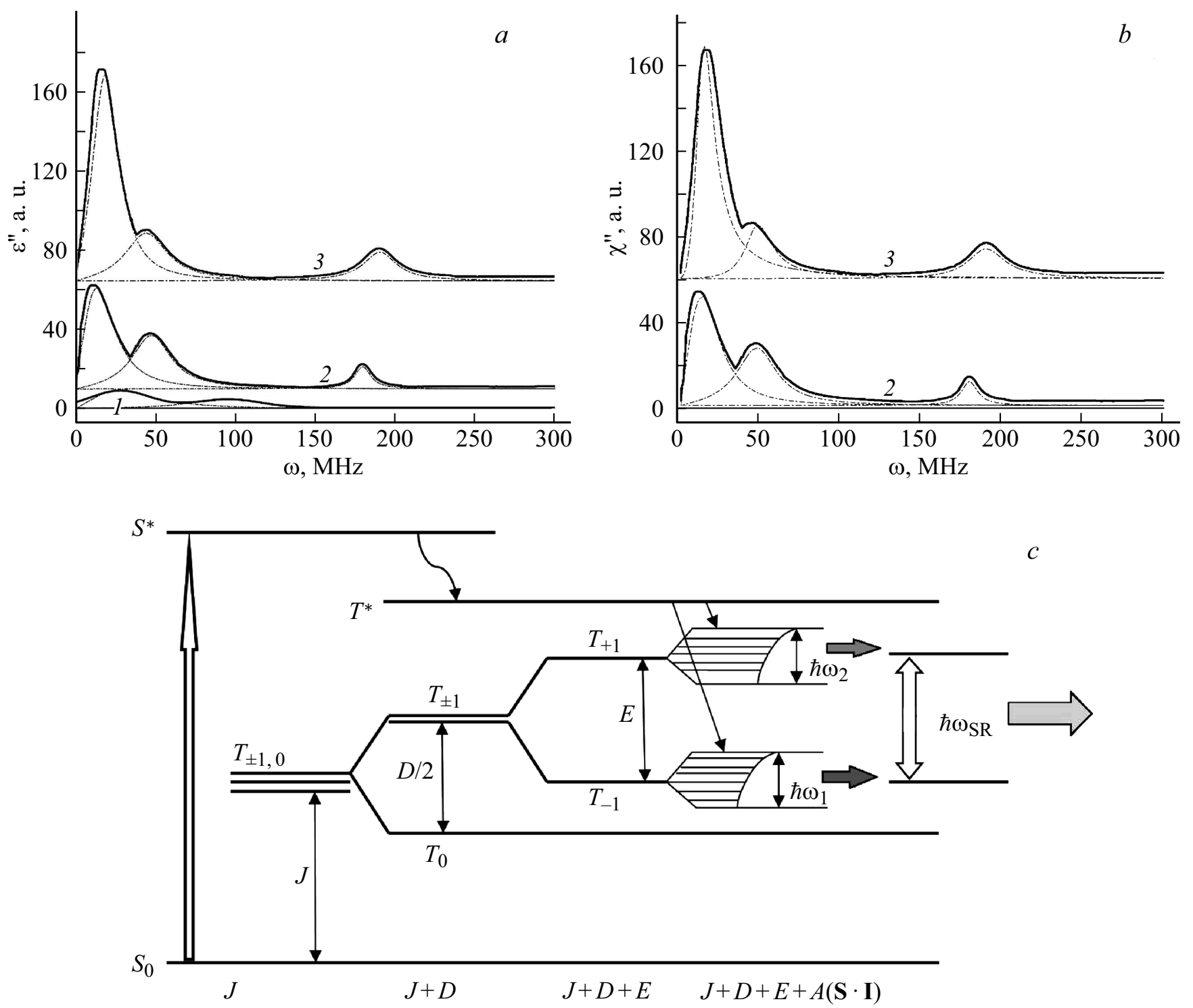

Рис. 2. $a$ - фурье-образы электрических сигналов PS-матрицы (1), PS-БК Со (2) и PS-БК Мn (3) и полосы поглощения, полученные по методу Лоренца; $b$ - фурье-образы электрических сигналов и полосы поглощения для PS-БК Со (2) и PS-БК $\mathrm{Mn}(3)$, полученные по методу ЛЛБ; $c$ - энергетическая диаграмма получения инверсии населенности уровней $T_{+1}$ и $T_{-1}$ (длина горизонтальных отрезков пропорциональна населенности уровней при расщеплении $T_{+1}$ и $T_{-1}$ по механизму спин-спинового взаимодействия).

(15.76) $\mathrm{mT}, \quad \Delta H_{a x}=16.59 \quad(20.52) \mathrm{mT}, \quad \Delta H_{a y}=0.87$ $(0.91) \mathrm{mT}, \Delta H_{a z}=0.69(0.89) \mathrm{mT}$. Для второго центра БК Со (или БК Мn) подобные параметры соответственно равны: $g_{b x}=2.1807$ (2.0158), $g_{b y}=2.1865$ (1.8974), $g_{b z}=2.2186$ (1.8847), $A_{b x}=4.91(5.43) \mathrm{mT}, A_{b y}=3.57$ (5.81) $\mathrm{mT}, A_{b z}=0.03$ (35.47) mT, $\Delta H_{b x}=2.24$ (3.41) mT, $\Delta H_{b y}=1.29(0.15) \mathrm{mT}, \Delta H_{b z}=5.83$ (2.42) $\mathrm{mT}$. Параметры расщепления в нулевом поле для БК Со (БК Мn) равны $D=4.90 \quad(19.48) \mathrm{mT} \quad$ и $\quad E=5.83 \quad(6.21) \mathrm{mT}$. Константы скалярного обменного взаимодействия равны $J=10.15(24.20) \mathrm{mT}$, а анизотропного обменного взаимодействия $G=43.98$ (23.06) $\mathrm{mT}$. Если константы скалярного и анизотропного обменных взаимодействий превышают величину дипольных констант, то это свиде- тельствует о косвенной природе спинового обмена [8], т.е. о том, что ионы кобальта и марганца связаны между собой, например, через кислородный анион. Таким образом, наиболее вероятно рассматриваемые спектры ЭПР можно соотнести с комплексами типа $\mathrm{Co}(\mathrm{QH})_{2}-\mathrm{O}-\mathrm{Co}(\mathrm{QH})_{2}$ и $\mathrm{Mn}(\mathrm{QH})_{2}-\mathrm{O}-\mathrm{Mn}(\mathrm{QH})_{2}$.

Подобные двухспиновые объекты обладают магнитноэлектрическим эффектом, который обусловлен парами неколлинеарных спинов, т.е. собственный электрический дипольный момент $\mathbf{D}$ связан с парой магнитных атомов с наклонными спинами $\mathbf{S}_{a}, \mathbf{S}_{b}$ и записывается в виде $\mathbf{D} \propto \mathbf{R} \times\left(\mathbf{S}_{a} \mathbf{S}_{b}\right)$, где $\mathbf{R}-$ относительное положение атомов [9]. Поскольку $\mathbf{R}$ изменяется во времени, соответственно изменяются векторы по- 
ляризации р и магнитные моменты системы М, которые связаны со спиновым ансамблем. Динамические спектры таких систем описываются в рамках модели Лоренца для гармонического осциллятора с затуханием $[10]$, а также в рамках модели слабого ферромагнетизма уравнением Ландау-Лифшица, модифицированным Бломбергеном (модель ЛЛБ $[11,12])$, т.е. уравнениями

$$
\begin{gathered}
\ddot{\mathbf{p}}+\alpha_{p} \dot{\mathbf{p}}+\omega_{R}^{2} \mathbf{p}=\varepsilon_{0} \omega_{R}^{2} \mathbf{E}(t), \\
\frac{d \mathbf{M}}{d t}=-\alpha_{m} \mathbf{M} \times \mathbf{H}+\omega_{r}\left[\chi_{0} \mathbf{H}-\mathbf{M}\right],
\end{gathered}
$$

где $\mathbf{p}, \mathbf{E}(t), \mathbf{M}, \mathbf{H}$ - векторы поляризации, осциллирующего электрического поля, магнитного момента, осциллирующего магнитного поля; $\omega_{R}$ и $\omega_{r}$ - частоты осцилляции поляризации и магнитного момента; $\alpha_{p}$ и $\alpha_{m}-$ константы поляризации и спин-поляризации; $\varepsilon_{0}$ и $\chi_{0}-$ электрическая постоянная и постоянная магнитной восприимчивости.

Как видно из рис. 2, $a$, фурье-образы сигналов $E \sim E(t) \sim J(t)$ имеют вид полосчатых спектров. Фурьеанализ импульсов $J(t) \sim E(t)$ показал, что наблюдаемые процессы СИ лежат в диапазоне частот от 0 до $200 \mathrm{MHz}$, а диапазон излучения (не СИ!) от контрольных образцов матричного полимера (полистирола) лежит в диапазоне от 0 до $100 \mathrm{MHz}$ (рис. 2,a). Высокочастотные колебания $E(t)$ связаны с колебаниями зарядов и, очевидно, обусловлены колебаниями поляризации. А поскольку изменения поляризации обусловлены системой диполей на основе ансамбля неколлинеарных спинов (системой димеров Дзялошинского-Мория, коими являются БК Со и БК $\mathrm{Mn}$ ), анализировать спектры для систем PS + БК Со или PS + БК Mn можно, используя приведенное для $\varepsilon^{\prime \prime}(\omega)$ уравнение (3) и приведенное для $\chi^{\prime \prime}(\omega)$ уравнение (4), т.е. по формулам

$$
\begin{aligned}
& \varepsilon^{\prime \prime}(\omega)=\varepsilon_{0} \frac{2 \alpha_{p} \omega_{R}^{2} \omega}{\left(\omega_{R, 0}^{2}-\omega^{2}\right)^{2}+4 \alpha_{p}^{2} \omega^{2}}, \\
& \chi^{\prime \prime}(\omega)=\chi_{0} \frac{2 \alpha_{m} \omega^{2} \omega_{r}}{\left(\omega_{r, 0}^{2}-\omega^{2}\right)^{2}+4 \omega^{2} \omega_{r}^{2}} .
\end{aligned}
$$

Как видно из рис. $2, a, b$, подобные аппроксимации дают хорошее согласие с экспериментом. Полученные выше данные позволяют утверждать, что за процессы СИ отвечает спиновый резервуар системы. Действительно, если постулировать, что накачка системы при РВ peaлизует быстрые процессы перехода БК Со и БК Мn из синглетного состояния $S$ в возбужденное синглетное состояние $S^{*}$, а затем в возбужденное триплетное состояние $T^{*}$, то дальнейший процесс можно описать, опираясь на полученные значения для констант взаимодействия $J, G, D, E$ (из формулы (2)), и построить диаграмму расщепления триплетных уровней $T_{+1}, T_{-1}$ и $T_{0}$ в магнитном поле (схема процесса приведена на рис. 2,c). При этом следует учесть, что для двухспиновых систем с ядрами металлов с $I \neq 0$ (в нашем случае это $I=9 / 2$ и 5/2 для ядерных спинов кобальта и марганца соответственно) происходит расщепление уровней $T_{+1}$ и $T_{-1}$ во внутреннем магнитном поле $E$ (равно константе спин-спинового взаимодействия из формулы (2)), а далее каждый из уровней $T_{+1}$ и $T_{-1}$ расщепляется на систему подуровней, между которыми разрешены квантовые переходы за счет снятия вырождения спин-спинового взаимодействия электронов и ядер при воздействии внутреннего магнитного поля $H_{i n t}$ (поле $H_{i n t}$ является функцией анизотропного обменного взаимодействия, $H_{i n t}=f(G)$ [3]). Между этими подуровнями могут реализоваться процессы электронных переходов, которые, вероятно, ответственны за низкочастотные полосы в диапазоне от 0 до $100 \mathrm{MHz}$. Процесс СИ - это реализация запрещенного перехода между уровнями $T_{+1}$ и $T_{-1}$, поскольку только расщепление $\Delta H=E$ (константа спинспинового взаимодействия из формулы (2)) для БК Со и БК $\mathrm{Mn}$, равное 5.83 (6.21) $\mathrm{mT}$, можно соотнести и сопоставить с полосами СИ при 180 и $189.7 \mathrm{MHz}$ $(1 \mathrm{mT} \sim 30 \mathrm{MHz})$.

Таким образом, в работе показана возможность реализации параметрического процесса радиочастотного сверхизлучения внешним механическим воздействием под высоким давлением на парамагнитные системы, образуемые неколлинеарными спинами.

\section{Финансирование работы}

Работа выполнена при поддержке Министерства науки и высшего образования РФ.

\section{Конфликт интересов}

Авторы заявляют, что у них нет конфликта интересов.

\section{Список литературы}

[1] Dicke R.H. // Phys. Rev. 1954. V. 93. P. 99-113.

[2] Dzyaloshinskii I.J. // Phys. Chem. Solids. 1958. V. 4. P. 241248.

[3] Moriya T. // Phys. Rev. 1960. V. 120. P. 91-99.

[4] Bridgman P.W. // Rev. Mod. Phys. 1946. V. 18. P. 1-37.

[5] Александров А.И., Александров И.А., Зезин С.Б., Дегтярев Е.Н., Дубинский А.А., Абрамчук С.С., Прокофьев А.И. // Хим. физика. 2016. Т. 35. № 2. С. 78-85. DOI: $10.1134 / \mathrm{S} 1990793116010139$

[6] Александров А.И., Шевченко В.Г., Александров И.А. // Высокомолекуляр. соединения А. 2018. Т. 60. № 1. С. 7682. DOI: $10.7868 / \mathrm{S} 2308112018010017$

[7] Абакумов А.Г., Черкасов В.К., Бубнов М.П. Эллерт О.Г. // Изв. АН. Сер. хим. 1992. № 10. С. 2315-2322. 
[8] Rakhimov R.R., Dobryakov S.N., Borisov Yu.A., Prokof'ev A.I., Aleksandrov A.I. // J. Chem. Phys. 2003. V. 118. P. 6017-6023. DOI: $10.1063 / 1.1556752$

[9] Kaplan T.A., Mahanti S.D. // Phys. Rev. B. 2011. V. 83.

P. 174432-174437. DOI: 10.1103/PhysRevB.83.174432

[10] Lorentz H.A. // Phys. Z. 1899. V. 498. P. 514-591.

[11] Landau L., Lifshitz L. // Phys. Z. Sow. 1935. V. 8. P. 153-165.

[12] Bloembergen N. // Phys. Rev. 1950. V. 78. P. 572-578. 